\title{
Development of Sealant Inspection System Using Image Processing with Reduced Effect of Ambient Light
}

\author{
Shingo Aramaki ${ }^{\mathrm{a}, *}$, Yuichi Hara ${ }^{\mathrm{b}}$, Yoshiyuki Daimaruc ${ }^{\mathrm{c}}$, Shota Nakashima ${ }^{\mathrm{a}}$, Kanya Tanaka ${ }^{\mathrm{a}}$, Atsushi Sanada ${ }^{\mathrm{d}}$ \\ ${ }^{a}$ Graduate School of Science and Engineering, Yamaguchi University \\ 2-16-1, Tokiwadai, Ube-city, Yamaguchi, 755-8611, Japan \\ besign Division, Hokusei Kogyo Corporation \\ 1-6-19, Kiyomizu, Kokurakita-ku, Kitakyushu-city, Fukuoka, 803-0841, Japan \\ 'Manufacturing Department, Nissan Motor Kyushu Co., Ltd. \\ 1-3, Shinhama-cho, Kanda-machi, Miyako-gun, Fukuoka, 800-0395, Japan \\ ${ }^{\mathrm{d}}$ Department of Integrated System Engineering, Nishinippon Institute of Technology \\ 1-11, Aratsu, Kanda-machi, Miyako-gun, Fukuoka, 800-0394, Japan \\ *Corresponding Author: u003vk@ yamaguchi-u.ac.jp
}

\begin{abstract}
In recent years, a variety of manufacturing is being developed. In the factory production line, an automatic inspection system with lower cost and higher precision is needed. Many inspection systems use image processing. However, generally the inspection system using image processing cannot be examined in certain optical condition, i.e., room light or sunlight can greatly affect the performance. Another problem is high cost for capital investment of the inspection system. In order to solve these problems, an image processing method using the $\mathrm{YCbCr}$ color space characteristics and cyan light is proposed. Also, an inexpensive system for its implementation is developed. Finally, the inexpensive image processing inspection system for reducing the influence of ambient light can be realized.

Keywords: Image processing, Sealant inspection system, YCbCr color space, Ambient light.

\section{Introduction}

In recent years, the variety of manufacturing is being developed. Users require the high quality of industrial product made from the process. It can improve the reliability of the manufacturer. In this way, the inspection process for the purpose of security of society and quality assurance of the product has been introduction into the production line of the factory ${ }^{(1)}$.
\end{abstract}

In the factory production line, the inspection process visual have been performed for a long time. However, in recent years, mass production of industrial products are required in the factory to meet the user demands. Therefore, rigor of standards for product quality, and in conjunction with the spread of total inspection, automation of the inspection process has become essential. Further, recently, the hardware such as the camera and the LED have high performance. At the same time, the price has been reduced. Therefore, many inspection processes have realized automation by using a camera ${ }^{(2-4)}$. Inspection process of sealant in Nissan Motor Kyushu plant that is covered in this research is automated.

This research has been conducted by the joint research of Nishinippon Institute of Technology and Nissan Motor Kyushu. At the moment, the automotive sealant that has been applied to the seam of iron plate of the car wheel parts is inspected on a production line of Nissan Motor Kyushu plant. In addition, this sealant is used in order to prevent the water leak from the outside of a car. The proper conditions at this time are shown as follows.

- Specified range within the coating width of the automotive sealant.

- There are no cracks in the automotive sealant.

The automotive sealant is inspected using image processing whether conditions are fulfilled and it is applied. 


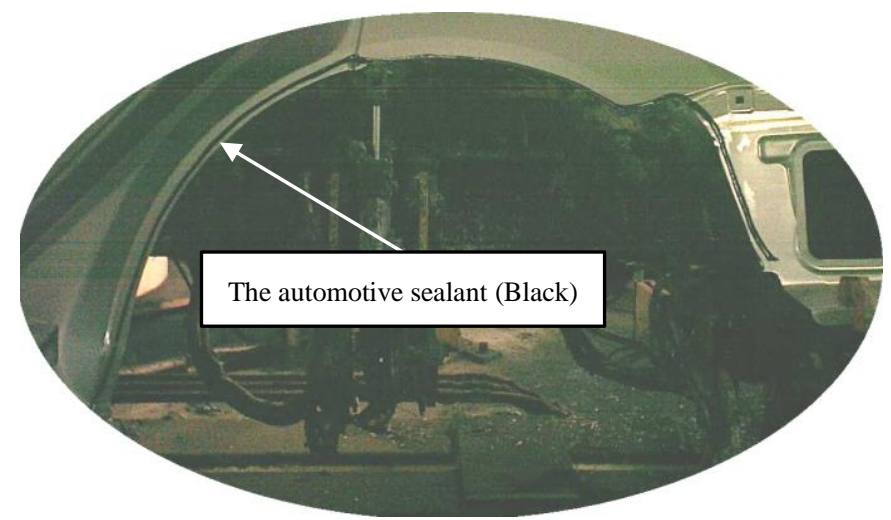

Fig. 1. The sealant at the time of the inspection.

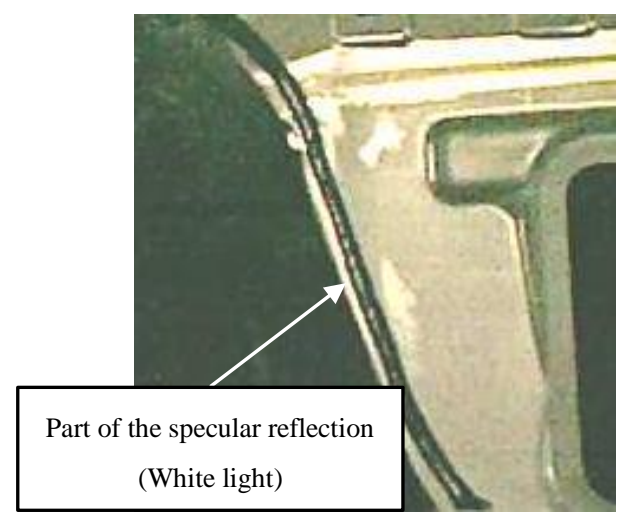

Fig. 2. Part of the specular reflection.

The picture of the automotive sealant to be examined to acquire by this inspection process is shown in Fig.1.

The following problems can be mentioned in the inspection system current.

(a) Specular reflection (white light) occurs on the sealant surface in response to the influence of various ambient light.(Fig.2)

(b) Capital investment cost of the license is expensive.

We describe the details of the above-mentioned problems. The first problem is that (a). Ambient light changes from moment to moment : outside light from the skylight, work light, and sputtering or the like is emitted always in the factory. Therefore, the specular reflection occurs on the sealant surface with high reflectivity. The image processing of this portion is difficult. There is a solution that can block the ambient light by covering with a box-shaped inspection system. However, the case studies covered in the present research, a large-scale introduction like this is very difficult. Therefore, it has become a problem. The second problem is that (b). The capital investment for the software license of image processing, i.e., about 2.81 million yen of introducing cost, about 1.56 million yen of horizontal development cost, is very expensive now. Thus, it is also a problem.

In this research, we aim to solve the above problems. We propose an optimal image processing inspection system with reduced specular reflection ${ }^{(5,6)}$ due to the influence of ambient light using an inexpensive system components.

\section{System Configuration}

High cost of capital investment of the license is a problem. In order to solve this problem, we have constructed an image processing inspection system using inexpensive system components.

First, the used software of the system components were free software freely available on the Internet. We used Microsoft Visual Studio Express 2012 for Windows Desktop as an integrated development environment. In addition, we used OpenCV2.4.3 as image processing library.

Subsequently, the used hardware of the system components were LED lighting and cameras. We used the web camera (i.e., LogicoolHDProWebcamC920) manufactured by Logicool as a camera for an experiment. On the other hand, we also used the network camera (i.e., acA2500-14gc) manufactured by Basler as a camera for an installation. The camera uses two types. As the history, the used web camera for the experiment is a very low cost. However, there is a problem, i.e., hard to get the same camera at the time of failure, because the change of a standard is intense. Moreover, since it is USB connection, there is a problem that the maximum transmission distance is short. We have used as a factory installed for the network camera to solve this problem. Finally, we used MEGA BAR LED RC manufactured by American DJ as LED lighting to illuminate the target object.

A system configuration diagram is shown in Fig.3 and Fig.4, where the solid line (— ${ }^{-}$indicates signal, and the dash line ( - - - ) indicates power supply. Then, round rectangle ( $\bigcirc$ ) indicates power source, the rectangle ( $\square$ ) indicates the device.

(a) System configuration for the experiment

The used system configuration as the experimental verification is described in Fig.3.

(b) System configuration for installation

The used system configuration as the field-installed sealants inspection process of Nissan Motor Kyushu plant is described in Fig.4. 


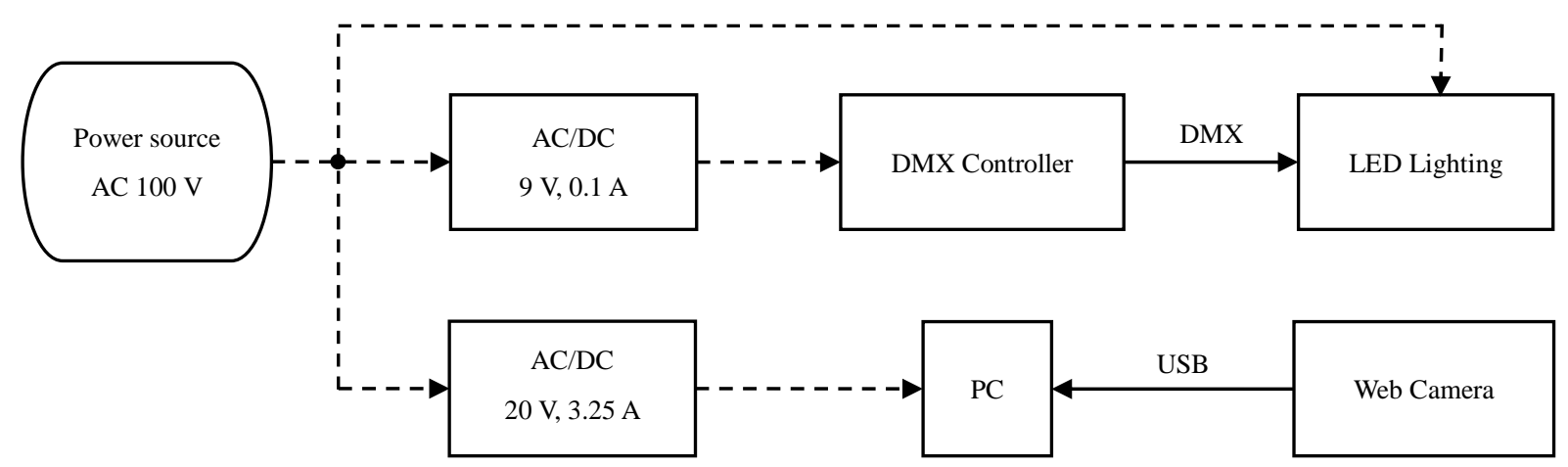

Fig. 3. The system configuration for the experiment.

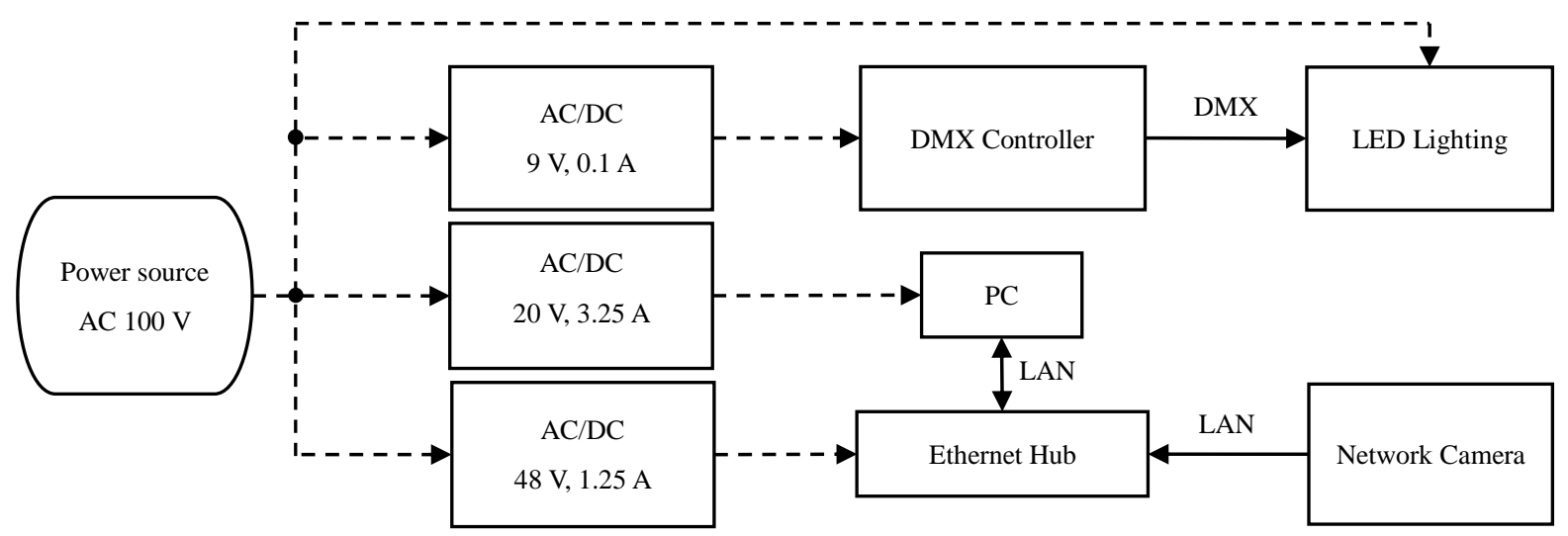

Fig. 4. The system configuration diagram for installation.

\section{YCbCr Color Space Characteristics}

\subsection{YCbCr Color Space}

$\mathrm{YCbCr}$ color space is the method of expressing a color by $\mathrm{Y}$ of a luminosity ingredient, and $\mathrm{Cb}(\mathrm{B}-\mathrm{Y})$ and $\mathrm{Cr}(\mathrm{R}-\mathrm{Y})$ of two color difference ingredients. This is also called YUV color space. Experimental results showed that human vision is generally sensitive to changes in brightness, but it is insensitive to changes in color.

There is property of the human eye that is sensitive to changes in brightness. However, it is insensitive to color change. Therefore, it is possible to reduce the color information of $\mathrm{Cb}$ and $\mathrm{Cr}$ components. In consequence, those are applied in various fields such as lossy compression technique or television broadcast ${ }^{(7-11)}$.

We focus on the $\mathrm{YCbCr}$ color space characteristics in this research. And, we have applied to the reduction of the specular reflection component and to the extraction of the sealant.

\subsection{Conversion to $\mathrm{YCbCr}$ Color Space from RGB Color Space}

An example of a conversion formula to $\mathrm{YCbCr}$ color space from the RGB color space is described in Eq.(1).

$$
\left\{\begin{aligned}
Y & =0.299 R+0.587 G+0.114 B \\
C b & =0.564(B-Y)+128 \\
C r & =0.713(R-Y)+128
\end{aligned}\right.
$$

From Eq.(1), conversion to the YCbCr color space can be performed from the RGB color space.

\subsection{Color Space Separation}

$\mathrm{YCbCr}$ color space or RGB color space is a multichannel image with three components. Color space separation is separated into only a single channel component from a specific multi-channel image.

We investigated $\mathrm{YCbCr}$ color space characteristics in this research. One obtained by the color space separation is described in Fig.5. 


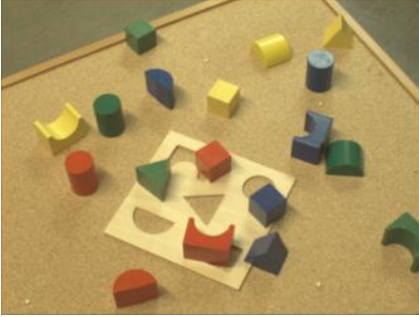

(a) Input image of the sample

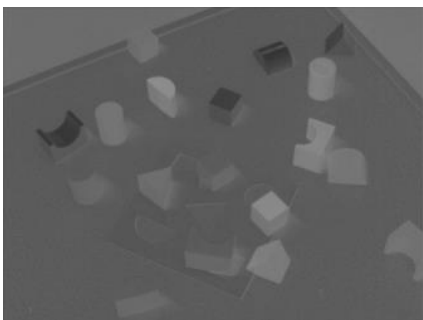

(c) Output image of the $\mathrm{Cb}$ channel

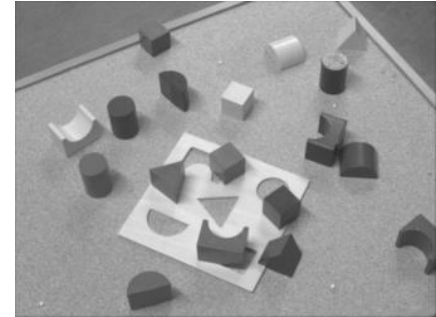

(b) Output image of the Y channel

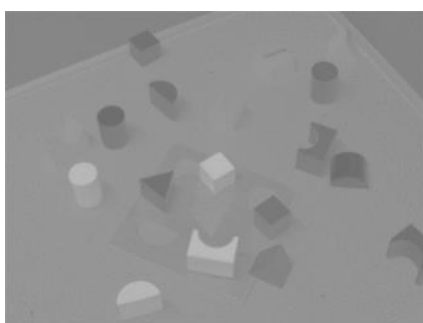

(d) Output image of the $\mathrm{Cr}$ channel

\subsection{Application and Consideration of $\mathrm{YCbCr}$ Color Space Characteristics}

We made a comparison verification of the four images in Fig.5. We compared Fig.5(a) with Fig.5(c). From this results, we found that $\mathrm{Cb}$ channel increase the brightness values of the blue component only. Then, we compared Fig.5(a) with Fig.5(d). From this results, we found that $\mathrm{Cr}$ channel increase the brightness values of the red component only.

As a result, region extraction of the red component and blue component is possible done by the $\mathrm{YCbCr}$ color space characteristics. In this research, we extract the sealant region of the test object by a combination of the $\mathrm{YCbCr}$ color space characteristics and the LED lighting of a specific wavelength. The specular reflection component due to the influence of the ambient light at the same time can be reduced.

\section{Experiment for Verification of the Property of Sealant}

We examined the characteristics of the sealant. In consequence, we have selected wavelength of optimal LED lighting. In addition, we have selected the best channel of $\mathrm{YCbCr}$ color space that suits it. Finally, it is confirmed by experiment to be useful in reducing the specular reflection component due to the influence of ambient light and extracting the sealant areas of the test object.

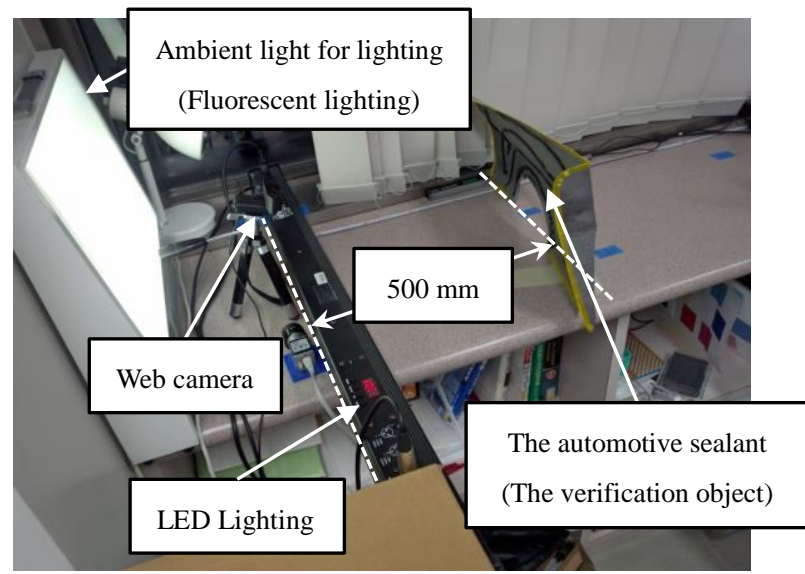

Fig. 6. Experimental apparatus.

\subsection{Experimental Condition}

We used the automotive sealant as a verification object in this experiment. And, the distance to the verification object was $500 \mathrm{~mm}$. Fig.6 shows the experimental device.

\subsection{Measurement Experimentation of $\mathrm{Cb}$ and $\mathrm{Cr}$}

The images were acquired verification object by web camera when illuminated LED lighting with a wavelength of various patterns. (Pattern of irradiation wavelength : OFF(extinction), green light, cyan light, blue light, violet light. This is except for the wavelength that is contained in the ambient light : red light, yellow light, white light. ) We have measured the $\mathrm{Cb}$ and $\mathrm{Cr}$ value of the iron plate portion and the sealant black portion by using the images. The comparisons were made using the measured data.

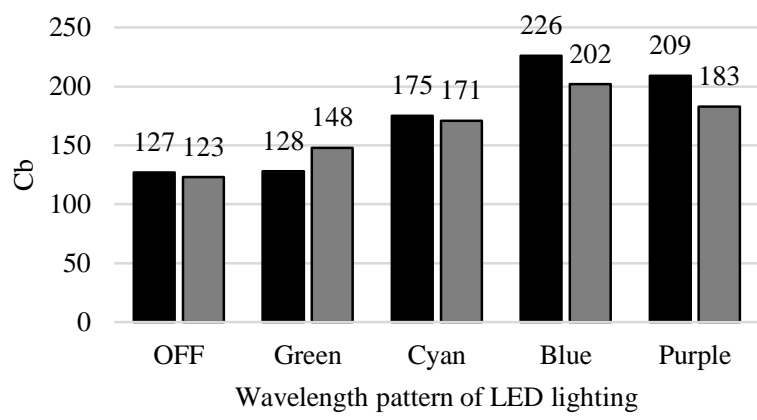

The black part of the automotive sealant $\square$ Part of iron plate

Fig. 7. Measurements of $\mathrm{Cb}$ using an LED lighting. 


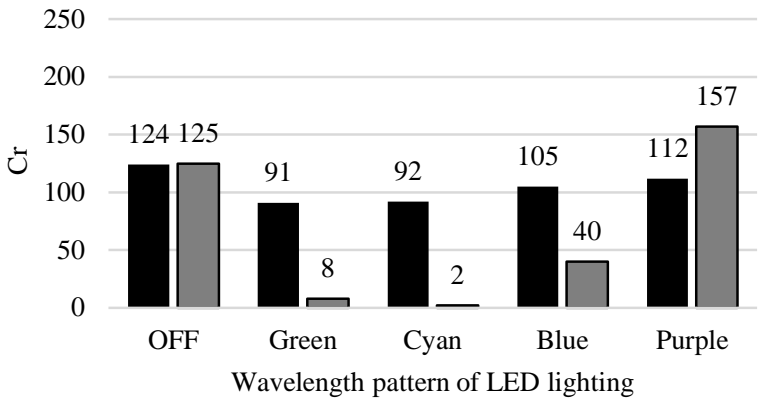

- The black part of the automotive sealant $\square$ Part of iron plate

Fig. 8. Measurements of $\mathrm{Cr}$ using an LED lighting.

We found that from the measurement results of Fig.7 and Fig. 8 , the difference between the Cr-value of the iron plate portion and the Cr-value of the sealant black portion is largest by cyan light irradiation. Therefore, it is possible to extract of the sealant when illuminated cyan light in $\mathrm{Cr}$ channel image.

\subsection{Experiment for Verification and Comparison of Cr Channel Image}

We compared the input image with $\mathrm{Cr}$ channel image obtained by illuminating the cyan light that is selected in Section 4.2. As a result, we confirmed the extraction of the sealant area. In addition, we also confirmed that some reduction of the specular reflection component due to the influence of ambient light can be. Figure 9 shows an input image of a cyan light irradiation. Figure 10 shows the $\mathrm{Cr}$ channel image of cyan light irradiation.

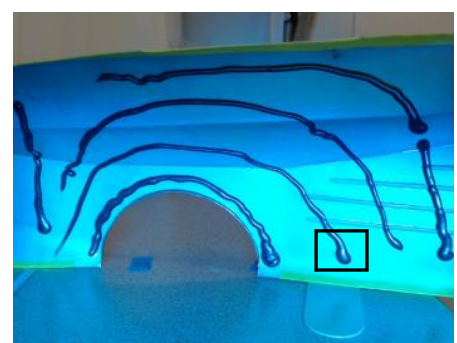

(a) Input image

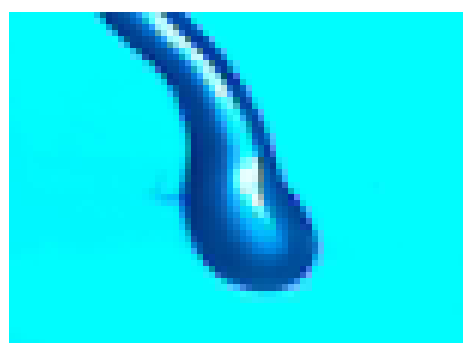

(b) Enlarged image of the square part
Fig. 9. Input image of a cyan light irradiation.

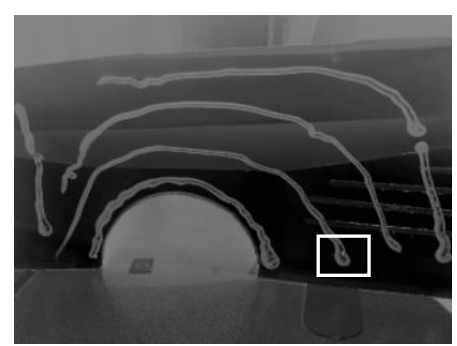

(a) Output image

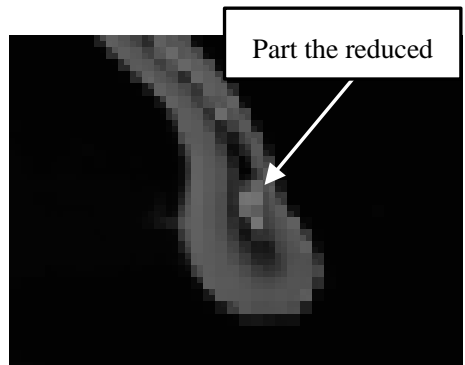

(b) Enlarged image of the square part
Fig. 10. The Cr channel image of cyan light irradiation.
Table 1. The number of pixels of the specular reflection component.

\begin{tabular}{|c|c|c|}
\hline Input image & Cr channel image & Reduction rate \\
\hline 155 pixel & 94 pixel & $39.4 \%$ \\
\hline
\end{tabular}

From these figures, it is possible to confirm reduction of specular reflection component, and extraction of the sealant area by $\mathrm{Cr}$ channel image of cyan light irradiation.

We compared the Cr channel image (Fig.10(b)) with the input image (Fig.9(b)) in cyan light irradiation. Thus, we determined the specular reflection component of the sealant surface using a discriminant analysis method ${ }^{(12,13)}$. Table 1 shows the number of pixels of the specular reflection component of the surface of the sealant. In addition, Table 1 shows reduction ratio of the specular reflection component.

From Table 1, the $\mathrm{Cr}$ channel images of cyan light irradiation was found that $39.4 \%$ of specular reflection components can be reduced. Finally, the usefulness of the $\mathrm{Cr}$ channel image of cyan light irradiation was confirmed.

\section{Conclusion}

It is possible to extract the sealant using the characteristics of the $\mathrm{Cr}$ channel and cyan light. Reduction of specular reflection due to influence of ambient light of the sealant was confirmed. Further, the area dividing the automotive sealant to define a threshold value for each area was verified. Therefore, we expected that the specular reflection can be removed completely.

\section{References}

(1) Morio Onoue, and other : "Automation of an inspection - Systematization handbook" , Fuji Techno System, 1990 (in Japanese)

(2) Yoji Marutani : "Automation technology of visual inspection”, Nikkan Kogyo Shimbun, Ltd., 1987 (in Japanese)

(3) Iwao Morishita, and other : "Automation of visual inspection", Institute of Electrical Engineers of Japan, 1984 (in Japanese)

(4) Elias N Malamas, Euripides G.M Petrakis, Michalis Zervakis, Laurent Petit, Jean-Didier Legat : "A survey on industrial vision systems, applications and tools", Image and Vision Computing, Vol. 21, No. 2, pp. 171188,2003

(5) Tomoaki Higo, Daisuke Miyazaki, Katsushi Ikeuchi : "Realtime Removal of Specular Reflection Component 
Based on Dichromatic Reflection Model", The Institute of Electronics, Information and Communication Engineers, Vol. 106, No. 230, pp. 77-84, 2006 (in Japanese)

(6) Robby T. Tan, Katsushi Ikeuchi : "Separating Reflection Components of Textured Surfaces Using a Single Image", IEEE Transactions on Pattern Analysis and Machine Intelligence, Vol. 27, No. 2, pp. 178-193, 2005

(7) Jen-Shiun Chiang, Chih-Hsien Hsia, Hao-Wei Peng, Chun-Hung Lien, Hsin-Ting Li : "Saturation adjustment method based on human vision with YCbCr color model characteristics and luminance changes", Intelligent Signal Processing and Communications Systems (ISPACS), pp. 136 - 141, 2012

(8) K.Shankar, K.Mahesh and Dr.K.Kuppusamy : “Analyzing Image Quality via Color Spaces", IJIPDV International Journal of Image Processing and Data Visualization, Vol. 1, No. 1, 2014

(9) Jose M. Chaves-González, Miguel A. Vega-Rodríguez, Juan A. Gómez-Pulido, Juan M. Sánchez-Pérez : "Detecting skin in face recognition systems: A colour spaces study”, Digital Signal Processing, Vol. 20, No.3, pp. 806-823, 2010

(10) Reza Azad, Hamidreza Shayegh Boroujeni : "Novel and Tunable Method for Skin Detection Based on Hybrid Color Space and Color Statistical Features", International Journal of Computer \& Information Technologies (IJOCIT), Vol. 1, No. 3, pp.211-219, 2013

(11) Mrs. Smriti Kumar, Mr. Deepak Singh : "Colorization of Gray Scale Images in YCbCr Color Space Using Texture Extraction and Luminance Mapping", IOSR Journal of Computer Engineering (IOSRJCE), Vol. 4, No. 5, pp. 27-32, 2012

(12) Nobuyuki Otsu, "An automatic threshold selection method based on the least-squares criterion and discrimination", Journal of the Institute of Electronics, Information and Communication Engineers, Vol.J63-D, No.4, pp. 349-356, 1980 (in Japanese)

(13) Nobuyuki Otsu, "A Threshold Selection Method from Gray-Level Histograms”, IEEE Trans. Systems Man, and Cybernetics, Vol. 9, No.1, pp. 62-66, 1979 